\title{
Research on Micro-power Wireless Meter Reading Based on IPv6 Zheng Tan, Yi-Fa MENG
}

\author{
Beijing Engineering Research Center of Security Alarm and Inspection
}

Beijing, China

\begin{abstract}
Keywords:power consumption information collection; IPv6 wireless communication; RPL routing protocol; micro-power wireless meter reading
\end{abstract}

\begin{abstract}
Because the number of electric industry end-users is tremendous and spot environment is complex, traditional meter reading pattern can't satisfy. In this paper, multicast routing algorithm based on IPv6 wireless communication module and RPL routing protocol is proposed. It is used to construct network in power consumption information collection system. What's more, networking meter reading flow of wireless sensor network based on IPv6 is designed, and it can copy from end to end. Finally, it is counted and analyzed for micro-power wireless copy pass rate of ten power supply companies in Beijing. It is demonstrated that this method is valid and feasible.
\end{abstract}

\section{Introduction}

With the popularization of smart grid all over the country, the requirements of electric management for data collection and transfer means are higher and higher, because power information collection system is a basic application system in smart power service system[1]. Meanwhile, it can guarantee grid operation safe and realize orderly electricity supply. The characteristics of micro-power wireless communication are high reliability, instantaneity and security[2]. Micro-power wireless communication has realized more than thousands of nodes application in state grid. It not only satisfies the requirement of remote meter reading basic service, but also becomes kernel support network of next generation marketing[3]. It effectively guarantees that the goal of state grid electric information collection system "all standing, all collection, all fee control" can be achieved. Presently, system operates well, the number of coverage users is more than 130000 and success rate of day reading data is above 99\%[4]. Therefore, to set more perfect wireless sensor network standard in electric information collection system is necessary[5].

\section{IPv6 Protocol}

IPv6 (Internet Protocol Version 6) is also named next internet protocol. IPv6 obviously improves quality of service, such as site capacity, security, administration of networks and mobility. It also provides useful character for smart object network. IP address space is expanded from 32 bit to 128 bit by IPv6, so the IP address is no longer limited. IPv6 no longer uses previous decimal notation, but uses new colon hexadecimal notation and compresses IPv6 address by 0 bit address compression method. With the increase of address digits, address can be divided into unicast, multicast and anycast address by IPv6. IPv6 hander is consisted of basic hander and extended hander. Extended hander can be extended neatly, and it is convenient to enlarge new option. Moreover, security of IPv6 obviously increased, so network layer of IPv6 can realize against resend attack and denial of service attack, prevent data session eavesdropping attacks and data passive or active listening. IPv6 can automatically deploy interface address and default router information for mainframe by neighbor discovery mechanisms. Therefore it can quickly set up link between internet and final user without user intervention .

Moreover IPv6 has obvious improvement in quality of mobile IP or Qos service. The advantages of IPv6 technology are as following:

1)The number of address is more, and every object has itself IP address;

2)It makes end-to-end data rate higher, and transmission speed faster; 
3)With well-determined IP protocol, it has stricter manage usage specification and can guarantee network smooth;

4)It can prevent virus or hacker from attacking, and network security is higher.

5)It can choose wired or wireless access way, and it makes deploy more convenient and faster.

These characteristics of IPv6 make it more useful in electric power industry. In electric power smart meter reading system, every electric device (such as terminal, concentrator, intelligent meter and collector) has an IP address. It simplifies software structure of meter reading network, and also is convenient to maintain personal work. Because devices can realize point-to-point communication, parameters and communication flows are decreased.

\section{Logic structure of electric information collection system networking}

The master station application program of electric information system can count, analyze or process user electric information within system and can provide decision-making level with gist. Network equipment, server and all kinds of administration terminals can satisfy the requirement of substratum each communication module. Master station should have timing collection, and conduct fixed point measure according to IPv6 address curtained by meter user. For example, it conducts real-time monitoring for meter electric energy data, anomalous event and electric quantity collection point.

Upstream interface of telecommunication module supports Ethernet, 3G and GPRS. Downstream interface of local communication module supports micro-power wireless. They are both IPv6 networks. Transmitted power of communication module should be less than $50 \mathrm{~mW}$. Time of transmitted duration should be less than $200 \mathrm{~ms}$. Received sensitivity is $-106 \mathrm{dbm}$. Error rate is $0.1 \%$. Modulation system is GFSK. Modulation frequency deviation is $20-30 \mathrm{kHz}$. Test traffic rate is 9600bit/s. communication between wireless module and electric meter uses serial asynchronous mode and MAC layer uses CSMA/CA conflict avoidance or automatic retransmission mechanism. According to Q/GDW 1376.2 code requirement, protocol of physical layer, MAC layer and data link layer makes frame structure and physical interface. Massive structure of electric information collection system is shown in Fig. 1.

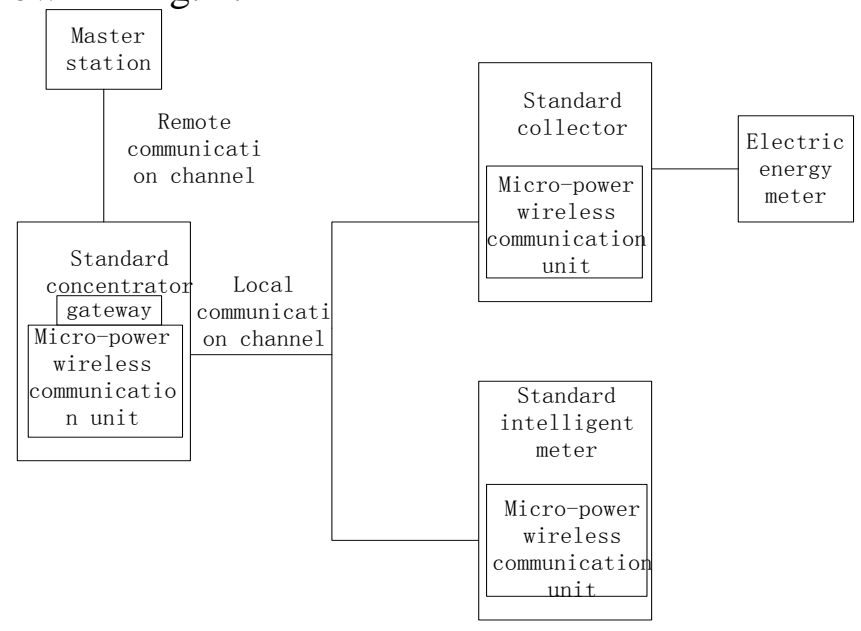

Fig. 1. Massive structure of electric information collection system

IPv6 communication device has UART interface, and makes it convenient that IEEE802.15.4g communication protocol attaches with other mainframe or embedded device (IEEE802.15.4g communication protocol is designed by working team according to new application (such as smart grid). It is a new physical layer standard that satisfies the requirement of low-cost, low power dissipation and long distance transmission. This standard is a supplement for IEEE802.15.4, and it greatly improves the capacity of resisting disturbance and compossibility. It obviously reduces power dissipation of intelligent instrument communication, and is helpful to improve stability and reliability of data transmission.). Therefore, collected data can be real-timely transmitted to upper computer protocol analysis software, and data is analyzed. 
Overall topology of IPv6 communication device is shown in Fig. 2.

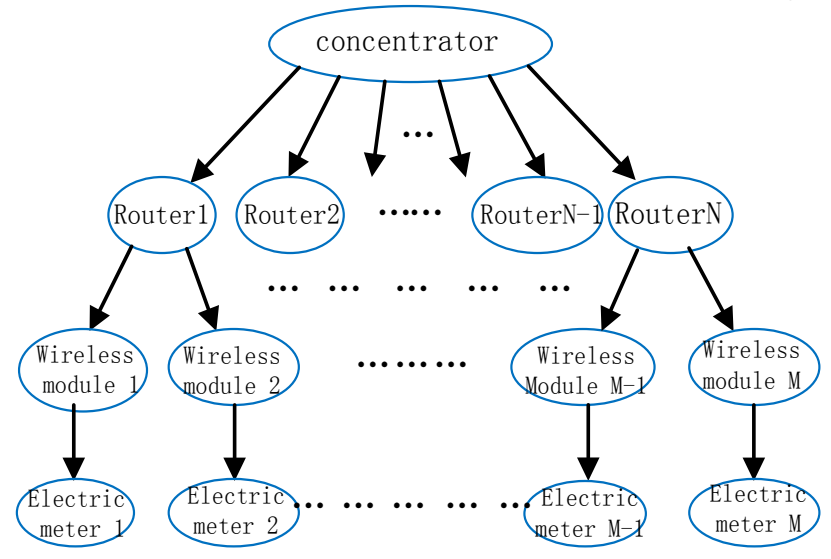

Fig. 2. Overall topology of IPv6 communication device

\section{Networking flow of meter reading based on IPv6 wireless sensor network}

According to configuration command of concentrator main reading module, micro-power wireless self-organized network that based on IPv6 is started. Concentrator sends out meter reading command after finishing network. It counts the number of objective IP addresses. If the number is one, it starts unicast routing meter reading based on optimal path value[11]. And meter reading instrument incessantly monitors 802.15.4 wireless signal strength instrument, it uses dynamic IPv6 routing protocol RPL (RFC6550). But if the node is invalid, routing will be converged and it will rebuild a new optimal path[12]; if path is invalid, routing will be converged to next optimal path. When finding objective path of many objective IP addresses, concentrator constructs a multicast meter reading down command frame of network layer based on IPv6 local micro-power wireless communication module[13]. Then, application layer data frames from concentrator are packaged in command frame. Finally, concentrator sends out multicast meter reading down command frame in form of multicast based on IPv6 local micro-power wireless communication module.

When micro-power wireless communication module based on IPv6 in electric energy meter receives down command frame, the objective IP address corresponding to frame number of extended MAC address is compared with multicast IP address. It judges handing information of multicast down command frame. If it has been processed, it was discarded. But it has not been processed, self IP address was compared with objective IP address of down command frame; if it is matching, objective IP address will be joined in a multicast group; if it is not matching, it conducts multicast transmission manage. Until finishing manage, micro-power wireless communication module sands out multicast meter reading termination command. Down data demand frame that is received by micro-power wireless module is sent to electric energy meter. If it doesn't receive response frame of electric energy meter within the required time, process will be stopped. If it receives response frame of electric energy meter within the required time, response data is sent to concentrator by network layer multicast meter reading up command of micro-power wireless module, and multicast meter reading is finished. The meter reading flow of automatic meter reading method based on IPv6 micro-power wireless module is shown in Fig. 3. 


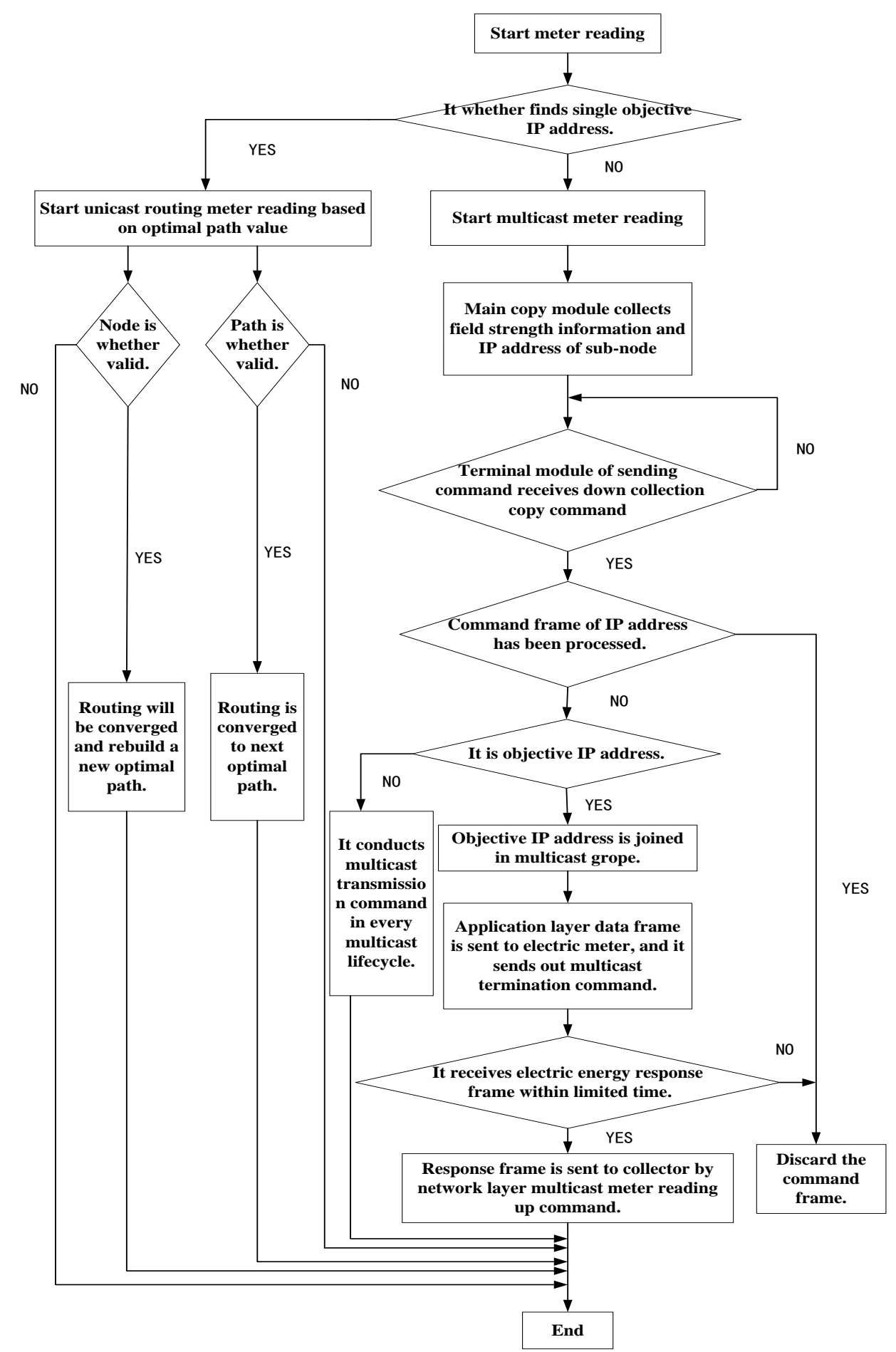

Fig. 3. The meter reading flow of automatic meter reading method based on IPv6 micro-power wireless module

\section{Networking copy pass rate of micro-power wireless in electric information collection system}

In Tab. 1, the number of collection terminals, the number of operation electric energy meters, and the number of copy pass electric energy meters in ten Beijing power supply companies are analyzed based on day and month copy pass rate. It is obvious that copy pass rates are all above $98 \%$, but it has a big difference from 0 packet loss probability in simulation. 


\begin{tabular}{cccccc}
\hline $\begin{array}{c}\text { the copy pass rate of } \\
\text { ten Beijing power } \\
\text { supply } \\
\begin{array}{c}\text { companiesPower } \\
\text { Supply Companies }\end{array}\end{array}$ & $\begin{array}{c}\text { the Number } \\
\text { of Collection } \\
\text { Terminal }\end{array}$ & $\begin{array}{c}\text { the Number of } \\
\text { Operation } \\
\text { Electric Energy } \\
\text { Meter }\end{array}$ & $\begin{array}{c}\text { the Number of } \\
\text { Copy Pass } \\
\text { Electric } \\
\text { Energy } \\
\text { Meter }\end{array}$ & $\begin{array}{c}\text { Daily } \\
\text { Copy } \\
\text { Pass Rate }\end{array}$ & $\begin{array}{l}\text { Monthly } \\
\text { Copy }\end{array}$ \\
\hline $\begin{array}{c}\text { Men-tou-gou } \\
\text { Power }\end{array}$ & 4248 & 110309 & 109616 & 99.37 & 99.36 \\
Fang-shan & 11717 & 305576 & 302704 & 99.06 & 99.08 \\
Da-xing & 17438 & 372701 & 370273 & 99.35 & 99.32 \\
Ping-gu & 8124 & 177547 & 176078 & 99.17 & 99.55 \\
Huai-rou & 6652 & 131095 & 129687 & 98.93 & 99.09 \\
Mi-yun & 7197 & 214363 & 213006 & 99.37 & 99.45 \\
Shun-yi & 7405 & 361552 & 355088 & 98.21 & 98.48 \\
Yan-qing & 4432 & 115390 & 114810 & 99.5 & 99.46 \\
Yi-zhuang & 1982 & 79880 & 78942 & 98.83 & 99.07 \\
Chang-ping & 12501 & 320560 & 315253 & 98.34 & 98.39 \\
\hline
\end{tabular}

\section{Conclusion}

A multicast routing method is proposed based on IPv6 wireless communication module and RPL routing protocol. And it is used to network in electric information collection system. Moreover, networking meter reading flow is designed based on IPv6 wireless sensor network. Finally, micropower wireless copy pass rate is analyzed for ten power supply companies in Beijing. It is demonstrated that this method is valid and feasible.

Although the problem of electric information collection based on IPv6 has been detailed studied in this paper and a multicast meter reading scheme is proposed, how to fuse interface protocol (Q/GDW1376.3-2013) with data transmission protocol (Q/GDW 1376.4-2013), function specification standard (Q/GDW1373-2013) and 6LoWPAN standard in electric information collection system, which needs to be further studied.

\section{Acknowledgment}

When implementation of the systems, the signals from the wireless sensor networks are preprocessed with the "SmartSignalProcessing" software developed by Prof. Ming-Yue ZHAI from North China Electric Power University.

\section{References}

[1] Pao-Hsiang His, Shi-Lin Chen. Distribution Automation Communication Infrastructure[J]. IEEE Transaction on Power Delivery,2008,V13(3):728-734.

[2] S.Amin, B.Wollenberg. Toward a smart grid [J]. IEEE Power and Energy Magazine, 2005, 31(3):34-41.

[3] Yu-Kai Huang, Yu-Kai Huang, Ai-Chun Pang, Ai-Chun Pang, Hui-Nien Hung, Hui-Nien Hung. A comprehensive analysis of low-power operation for beacon-enabled IEEE 802.15.4 wireless networks[J]. IEEE Transactions on Wireless Communications, 2009,8(11): 5601-5611.

[4] Jara Antonio, Silva Ricardo, Silva Jorge, Zamora Miguel, Skarmeta Antonio. Mobile IP-Based Protocol for Wireless Personal Area Networks in Critical Environments[J]. Wireless Personal Communications, 2011, 61(4): 711-737.

[5] Djamaa Badis, Richardson Mark, Aouf Nabil, Walters Bob. Towards efficient distributed service discovery in low-power and lossy networks[J].Wireless Networks, 2014,20(8):24372453. 
[6] Kermajani Hamidreza, Gómez Carles. Contributions to the performance evaluation and improvement of the IPv6 routing protocol for low-power and lossy networks[D]. Universitat Politècnica De Catalunya, 2014.

[7] Rawat Priyanka, Singh Kamal, Chaouchi Hakima, Bonnin Jean. Wireless sensor networks: a survey on recent developments and potential synergies[J].The Journal of Supercomputing, 2014,68(1):1-48.

[8] Tillison Joe.What's driving the success of IEEE 802.15.4 radios Low cost, sophistication, and relative ease of use are some of the key factors behind the standard's seemingly ubiquitous presence among short-range radio and wireless sensor-network designs[J].Electronic Design,2011, 59(5):61-65.

[9] ZHAI Ming-Yue, Transmission Characteristics of Low-Voltage Distribution Networks in China Under the Smart Grids Environment. IEEE Transaction on Power Delivery. Vol. 26, No. 1, JANUARY 2011, pp.173-180.

[10] Zhiqiang Xu, Mingyue Zhai, and Xiang Cui. Cross-Layer Optimization of User Scheduling and Resource Allocation in Power-line Communication Systems. IEEE Trans. on Power Delevery. Vol.26, No.3, 2011:1449-1458.

[11] Yan-Hong ZHAO, Jie TONG, Ming-Yue ZHAI, Dan LI. Application of IPV6 wireless sensor network on advanced measuring system for smart grid. Applied Mechanics and Materials, v 602-605, p 2426-2430, 2014, Advanced Manufacturing and Information Engineering, Intelligent Instrumentation and Industry Development.

[12] XING Gui-Lan, ZHAI Ming-Yue, SUN Xiao-Da, "Self-similarity of Traffic in Wireless Sensor Networks for Smart Grid", IJACT: International Journal of Advancements in Computing Technology, Vol. 5, No. 8, pp. $524 \sim$ 531, 2013

[13] JIA SHu-Guang, LU Li-Peng, SU Ling-Dong, XING Gui-Lan, ZHAI Ming-Yue. An Efficient Sleeping Scheduling for Save Energy Consumption in Wireless Sensor Networks. Advanced Materials Research. Vol.756-759, p 2288-2293. 2013 